\title{
Parte I. Requerimientos básicos de infectología para hospitales que atienden pacientes hemato-oncológicos: ambiente hospitalario, protocolos diagnósticos y arsenal terapéutico. Fundamentación
}

\author{
Ricardo Rabagliati', María Elena Santolaya², Marcela Ferres³, Marcela Rabello ${ }^{4}$ y Paula Catalán ${ }^{5}$
}

Basic requirements of facilities for hospitals that treat hemato-oncological patients: hospital environment, diagnostic protocols and therapeutic arsenal

The care of cancer patients, including recipients of hematopoietic stem cell transplantation, has numerous challenges for hospitals that must provide safe environments in which exposure to pathogens that generate morbidity and mortality is reduced at maximum. At the same time, they must have established protocols that allow a rational study of the possible infectious etiologies and the existence of an adequate therapeutic arsenal together with timely treatment algorithms, updated according to consensus guidelines and effective according to the suspected or confirmed infection. This article introduces some of the arguments that support these requirements, then that are developed in three successive articles dedicated to the hospital environment, diagnostic protocols and therapeutic arsenal.

Keywords: Cancer; hematopoietic stem cell transplantation; infection; hospital environment; quality \& safety.

Palabras clave: Cáncer; trasplante de precursores hematopoyéticos; infección; ambiente hospitalario; calidad y seguridad.

\section{Abreviaturas \\ EFI : enfermedad fúngica invasora \\ EICH : enfermedad de injerto contra hospedero \\ $|\mathrm{B}| \quad$ : infección bacteriana invasora \\ PHO : pacientes hemato-oncológicos \\ TPH : trasplante de precursores hematopoyéticos}

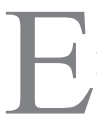

1 manejo integral del paciente con cáncer implica hacerse cargo de numerosos desafíos, tanto para sus equipos tratantes como para la institución de salud que los recibe para su tratamiento. Una de las mayores preocupaciones es el alto riesgo de infecciones que agregan morbilidad, significan alto costo económico y riesgo de mortalidad ${ }^{1,2}$, por lo que su prevención, diagnóstico oportuno y terapia efectiva son importantes materias que requieren constante revisión.

La frecuencia de infecciones en los pacientes hematooncológicos (PHO), incluyendo los que requieren trasplante de precursores hematopoyéticos (TPH), depende de varios factores, entre los que destacan el tipo de patología oncológica ${ }^{3}$, la edad, co-morbilidades asociadas y tipo de quimioterapia. En la medida que la neutropenia secundaria resulte más profunda y prolongada, mayor es el riesgo de infecciones bacterianas invasoras (IBI) y enfermedades fúngicas invasoras $(\mathrm{EFI})^{4-10}$. El TPH en sus distintas modalidades es parte del tratamiento de algunos cánceres hematológicos, terapia que implica largos períodos de neutropenia profunda que coinciden con el período de mayor exposición al ambiente hospitalario. Posteriormente, la enfermedad de injerto contra hospedero (EICH) y el uso de inmunosupresores para su manejo, también conllevan a un mayor riesgo de infecciones debido a la depresión de la inmunidad celular predominando infecciones oportunistas, en las que el ambiente también puede jugar un rol importante como factor de riesgo para una colonización e infección ${ }^{11-14}$.

Es conocido que las fuentes de infección de los PHO pueden ser endógena, es decir, de la propia microbiota del paciente, o exógena, que incluye el ambiente hospitalario y ambulatorio, donde la contaminación microbiológica del aire y agua, junto al entorno de personas que rodea a los pacientes, pueden significar un riesgo de infección. Múltiples reportes en la literatura médica dan cuenta del impacto de la exposición ambiental como factor de riesgo de infecciones, especialmente $\mathrm{EFI}^{15-19}$.

En la actualidad en nuestro país, la regulación vigente de infraestructura, organización, equipamiento y recursos humanos se encuentra determinada por el Decreto Supremo 161/82 de Autorización Sanitarias
'Departamento de Enfermedades Infecciosas del Adulto. Escuela de Medicina. Pontifica Universidad Católica de Chile. Santiago, Chile. Departamento de Pediatría. Hospital Dr. Luis Calvo Mackenna, Facultad de Medicina, Universidad de Chile. Santiago, Chile.

${ }^{3}$ Departamento de Enfermedades Infecciosas e Inmunología Pediátrica. Escuela de Medicina. Pontificia Universidad Católica de Chile. Escuela de Medicina. Pontificia Universidad Católica de Chile. Santiago, Chile. ${ }^{4}$ Unidad de Infectología, Hospital Dr. Luis Calvo Mackenna. Santiago, Chile. sUnidad de Trasplante de Médula ósea. Hospital Luis Calvo Mackenna. Santiago, Chile.

Los autores declaran ausencia de conflicto de interés. El presente trabajo no recibió financiamiento alguno.

Correspondencia a: Ricardo Rabagliati rabagli@med.puc.cl 
de Hospitales y Clínicas $^{20}$, que se complementa con un reglamento específico más recientemente publicado, que define estándares para los centros que administran quimioterapia ${ }^{21}$. Adicionalmente, existen publicaciones oficiales con recomendaciones del manejo ambiental para los centros que atienden pacientes que reciben $\mathrm{TPH}^{22}$, junto a normas de prevención de infecciones asociadas a aquellas actividades que generan polvo ambiental en el ambiente hospitalario ${ }^{23}$. Por otra parte, dentro del marco regulatorio vigente, la calidad se certifica a través del sistema nacional de acreditación ${ }^{24}$, el que no desarrolla en mayor profundidad estándares específicos de seguridad relacionados a los procesos de prevención, atención, diagnóstico y de terapia de infecciones de pacientes con cáncer en sus distintas etapas de tratamiento, incluido el TPH.

Considerados todos estos aspectos, a nuestro juicio existe espacio para definir estándares referidos a infraestructura, diseño y suministro de servicios básicos de los establecimientos que atienden pacientes con cáncer y TPH, así como la definición de procesos seguros diseñados para aminorar los riesgos a que se exponen los pacientes en el ambiente hospitalario, los cuidados de transición al domicilio, las herramientas diagnósticas de laboratorio y el necesario arsenal terapéutico antimicrobiano de rápido acceso para el oportuno tratamiento de las complicaciones infecciosas en estos enfermos. El avance en la definición de estos estándares desde la perspectiva de las infecciones, puede contribuir a la creación en un futuro cercano de procesos de acreditación específicos de los centros que atienden pacientes oncológicos, incluyendo $\mathrm{TPH}$, para garantizar su atención con la mayor seguridad clínica. Existen interesantes ejemplos en la literatura médica internacional que han reportado cómo la implementación de programas de acreditación de centros de TPH se relaciona con mayor sobrevida de los pacientes ${ }^{25-28}$.

El objetivo de este trabajo fue entregar a infectólogos, microbiólogos, oncólogos, hematólogos, equipo clínicos $\mathrm{y}$ directivos de hospitales públicos $\mathrm{y}$ privados de Chile y de otros países de la región, una mirada integral de los puntos más relevantes para incrementar la seguridad clínica de los centros hospitalarios que atienden pacientes con cáncer y receptores de TPH.

Para cumplir este objetivo se presenta el documento en tres capítulos: el primero dedicado a Aspectos del Ambiente Hospitalario, incluyendo tópicos de diseño e infraestructura, procesos asistenciales seleccionados, calidad microbiológica del aire y agua, orientados a disminuir la potencial exposición a patógenos; el segundo a las Herramientas de Laboratorio para el diagnóstico etiológico de los síndromes clínicos más frecuentes que deberían guiar la evaluación de los pacientes, asegurando el completo estudio de las posibles etiologías infecciosas y el tercero, a Algoritmos Terapéuticos que estandaricen el manejo de infecciones seleccionadas por gravedad y/o frecuencia.

\section{Resumen}

La atención de pacientes con cáncer, incluyendo los receptores de trasplantes de precursores hematopoyéticos, plantea numerosos desafíos para los hospitales que deben proveer ambientes seguros, en que se logre aminorar al máximo posible la exposición a patógenos que generan morbilidad y mortalidad. Al mismo tiempo deben contar con protocolos establecidos que permitan realizar un estudio racional de las posibles etiologías infecciosas que pueden presentar estos pacientes. A su vez, deben asegurar la existencia de un arsenal terapéutico adecuado, junto a algoritmos de tratamiento oportuno, actualizado según guías consensuadas y efectivo según la infección sospechada o confirmada. En este artículo se introducen algunos de los argumentos que sustentan estos requerimientos que luego se desarrollan en tres artículos sucesivos dedicados al ambiente hospitalario, protocolos diagnósticos y arsenal terapéutico.

\section{Referencias bibliográficas}

1.- Kuderer N M, Dale D C, Crawford J, Cosler L E, Lyman G H. Mortality, morbidity, and cost associated with febrile neutropenia in adult cancer patients. Cancer 2006; 106: 2258-66. DOI: $10.1002 /$ cncr.21847.

2.- Maschmeyer G, Haas A. The epidemiology and treatment of infections in cancer patients. Int $\mathrm{J}$ Antimicrob Agents 2008; 3: 193-7. DOI: 10.1016/j.ijantimicag.2007.06.014.

3.- Klastersky J. Management of fever in neutropenic patients with different risks of complications. Clin Infect Dis 2004; 39 Suppl 1: S32-7. DOI: $10.1086 / 383050$.
4.- Bodey G P, Buckley M, Sathe Y S, Freireich E J. Quantitative relationships between circulating leukocytes and infection in patients with acute leukemia Ann Intern Med 1966; 64: 328-40. PMID: 5216294.

5.- Bow E J. Infection in neutropenic patients with cancer. Crit Care Clin 2013; 29 (3): 411-41. DOI: $10.1016 /$ j.ccc.2013.03.002

6.- Crawford J, Dale D C, Lyman G H. Chemotherapy-induced neutropenia: risk, consequences, and new directions for its management. Cancer 2004; 100 (2): 228-37. DOI: $10.1002 /$ cncr.11882.

7.- Santolaya M E, Álvarez A M, Becker A, Cofré J, Enríquez N, O`Ryan M, et al. Prospective, multicenter evaluation of risk factors associated with invasive bacterial infection in children with cancer, neutropenia, and fever. J Clin Oncol 2001; 19 (14): 3415-21. DOI: 10.1200/ JCO.2001.19.14.3415.

8.- Rabagliati B R, Fuentes L G, Orellana U E, Oporto C J, Domínguez M I, Benítez G R, et al. Etiología de episodios de neutropenia febril en pacientes adultos con cáncer hematológico y de órganos sólidos en el Hospital Clínico Universidad Católica, Santiago Chile. Rev Chil Infectol 2009; 26 (2): 106-13. DOI: /S071610182009000200001.

9.- Castagnola E, Fontana V, Caviglia I, Caruso S, Faraci M, Fioredda F, et al. A prospective study 
on the epidemiology of febrile episodes during chemotherapy-induced neutropenia in children with cancer or after hemopoietic stem cell transplantation. Clin Infect Dis 2007; 45 (10): 1296-304. DOI: 10.1086/522533.

10.- Castagnola E, Garrè M L, Bertoluzzo L, Pignatelli M, Pavanello M, Cayiglia I, et al. Epidemiology of febrile neutropenia in children with central nervous system tumor: results from a single center prospective study. J Pediatr Hematol Oncol 2011; 33 (7): e310-5. DOI: 10.1097/MPH.0b013e31822bf6ec.

11.- Srinivasan A, McLaughlin L, Wang C, Srivastava D K, Shook D R, Leung W, et al. Early infections after autologous hematopoietic stem cell transplantation in children and adolescents: the St. Jude experience. Transpl Infect Dis. 2014; 16 (1): 90-7. DOI: 10.1111/ tid. 12165.

12.- Mendes E T, Dulley F, Basso M, Batista M V, Coracin F, Guimaraes T, et al. Healthcareassociated infection in hematopoietic stem cell transplantation patients: risk factors and impact on outcome. Int J Infect Dis 2012; 16 (6): e4248. DOI: 10.1016/j.ijid.2012.01.015.

13.- Laws H J, Kobbe G, Dilloo D, Dettenkofer M, Meisel R, Geisel R, et al. Surveillance of nosocomial infections in paediatric recipients of bone marrow or peripheral blood stem cell transplantation during neutropenia, compared with adult recipients. Hosp Infect 2006; 62 (1): 80-8. DOI: 10.1016/j.jhin.2005.05.010.

14.- Marena C, Zecca M, Carenini M L, Bruschi A, Bassi M L, Olivieri P, et al. Incidence of, and risk factors for, nosocomial infections among hematopoietic stem cell transplantation recipients, with impact on procedure-related mortality. Infect Control Hosp Epidemiol 2001; 22 (8): 510-7. DOI: 10.1086/501942.

15.- Alberti C, Bouakline A, Ribaud P, Lacroix C, Rousselot P, Leblanc T, et al; Aspergillus Study Group. Relationship between environmental fungal contamination and the incidence of invasive aspergillosis in haematology patients.
J Hosp Infect. 2001; 48 (3): 198-206. DOI: 10.1053/jhin.2001.0998.

16.- Perdelli F, Cristina M L, Sartini M, Spagnolo A M, Dallera M, Ottria G, et al. Fungal contamination in hospital environments. Infect Control Hosp Epidemiol 2006; 27: 44-7. DOI: 10.1086/499149.

17.- Panagopoulou P, Filioti J, Petrikkos G, Giakouppi P, Anatoliotaki M, Farmaki E, et al. Environmental surveillance of filamentous fungi in three tertiary care hospitals in Greece. J Hosp Infect. 2002; 52: 185-91. PMID: 12419271

18.- Anaissie E J, Stratton S L, Dignani M C, Summerbell R C, Rex J H, Monson T P, et al. Pathogenic Aspergillus species recovered from a hospital water system: a 3-year prospective study. Clin Infect Dis 2002; 34 (6): 780-9. PMID: 11850861.

19.- Mesquita-Rocha S, Godoy-Martínez P C, Gonçalves S S, Urrutia M D, Carlesse F, Seber A, et al. The water supply system as a potential source of fungal infection in paediatric haematopoietic stem cell units. BMC Infect Dis 2013; 13: 289. DOI: 10.1186/1471-2334-13-289.

20.- Normas técnicas básicas para establecimientos de salud de atención cerrada. Ministerio de Salud. Disponible en: http://webminsal.cl/portal/url/item/ b0226a5e50cdb42fe04001011e0147fa.pdf.

21.- Reglamento sobre establecimientos de administración de quimioterapia oncológica. Subsecretaria de Salud Pública. Ministerio de Salud. Noviembre de 2014. Disponible en: http://www.diariooficial.interior.gob.cl/ media/2014/11/17/do-20141117.pdf.

22.- Revisión científica y recomendaciones para el manejo ambiental de pacientes trasplantados de médula ósea. Serie Cuaderno de Redes, $N^{\circ}$ 21. 2008. Subsecretaría de Redes Asistenciales. División de Integración de Redes. Departamento de Calidad y Seguridad del Paciente. Unidad de Evaluación de Tecnologías Sanitarias. Ministerio de
Salud. Gobierno de Chile. Disponible en: http://web.minsal.cl/portal/url/item/ aleb519e7e794592e04001011f01798b.pdf.

23.- Normas de prevención de infecciones asociadas a modificaciones estructurales y otras actividades que generan polvo ambiental en establecimientos hospitalarios. Circular $N^{0} 7.2$ de mayo de 2011. Subsecretaría de Redes Asistenciales. Departamento de Calidad y Seguridad del Paciente. Ministerio de Salud. Gobierno de Chile. Disponible en: http://web.minsal.cl/portal/url/item/ a48536280e55e9f4e04001011e0163bc.pdf.

24.- Manual del estándar general de acreditación para prestadores institucionales de atención cerrada. Superintendencia de Salud. Gobierno de Chile. Disponible en: http://www.supersalud. gob.cl/portal/articles-4530_Manual_AC_pdf. pdf.

25.- Chabannon C, Pamphilon D, Vermylen C, Gratwohl A, Niederwieser D, McGrath E, et al. Ten years alter the first inspection of a candidate European centre, an EBMT registry analysis suggest that clinical outcome is improved when hematopoietic SCT is performed in a JACIE accredited program. Bone Marrow Trasplant 2012; 47: 15-7. DOI: $10.1038 / \mathrm{bmt} .2011 .3$

26.- Pamphilon D, Apperley J F, Samson D, SlaperCortenbach I, McGrath E. JACIE accreditation in 2008: demonstrating excellence in stem cell transplantation. Hematol Oncol Stem Cell Ther 2009; 2: 311-9. DOI: 10.1016/S16583876(09)50019-2.

27.- LeMaistre CF, Loberiza FR Jr. What is quality in a transplant program? Biol Blood Marrow Transplant 2005; 11: 241-6 DOI: 10.1016/j. bbmt.2004.12.329.

28.- Gratwohl A, Brand R, Niederwieser D, Baldomero H, Chabannon C, Cornelissen J, et al. Introduction of a quality management system and outcome after hematopoietic stemcell transplantation. J Clin Oncol 2011; 29: 1980-6. DOI: 10.1200/JCO.2010.30.4121. 